\title{
Energy influx measurements with an active thermal probe in plasma-technological processes
}

\author{
Ruben Wiese ${ }^{1 *}$, Holger Kersten ${ }^{1}$, Georg Wiese ${ }^{2}$ and René Bartsch²
}

\author{
* Correspondence: wiese@physik. \\ uni-kiel.de \\ ${ }^{1}$ Institut für Experimentelle und \\ Angewandte Physik, Leibnizstr. 19, \\ Kiel D-24098, Germany \\ Full list of author information is \\ available at the end of the article
}

\begin{abstract}
Many plasma-technological applications are based on plasma wall interaction, which can be characterised by calorimetric probes to measure the energy influx from the plasma to the substrate surface. Passive probes are based on the principle of recording the temperature course during heating and cooling of the probe for calculating the energy influx. The disadvantages of these probes are that the energy influx has to be interrupted by switching off the energy source or by using suitable apertures and by the necessity of knowing the exact heat capacity of the probe.

A continuously operating active probe is, therefore, developed which does not need to be calibrated and which compensates the environmental effects as well as the heat conduction by the probe holder. By means of controlled electrical heating the probe is set to a given working temperature and then the energy supply supporting the fixed operating temperature is measured. The energy influx by the plasma is compensated by decreasing the heating power and is directly displayed in $\mathrm{J} / \mathrm{cm}^{2} \mathrm{~s}$. Some practical measurements are presented. Even, if the probe is designed as double probe the directionality of the energy influx can be determined.
\end{abstract}

Keywords: Sensors; Thermal probe; Energy influx; Process control

\section{Introduction}

A large variety of plasma-technological applications are based on plasma wall interaction which occurs via the generated plasma sheath. A good possibility for its characterisation is offered by calorimetric thermal probes for energy influx measurements from the plasma to the substrate, e.g. for the measurement of the deposited power.

Passive thermal probes are often used by several groups, based on the principle of recording the temperature temporal course at the heating and the cooling phase to calculate the energy influx [1,2]. A disadvantage of such passive probes is that the energy influx has to be interrupted by switching off the energy source or by using suitable apertures.

Active probes are also established, constructed as a planar substrate or as a rotationallysymmetric membrane, whereby the energy influx is determined by the measurement of a temperature gradient [3-5]. However, the temperature of these probes is not freely adjustable and its distribution along the surface is not constant. A calibration is necessary before the measurement which may constitute an additional error source.

A novel continuously operating active probe is presented which does not need to be calibrated and which compensates the environmental effects as well as the heat conduction by the probe holder. The change in the heat capacity of the probe by thin 
film deposition also does not influence the measured energy influx. With a double probe configuration it is even possible to measure the directionality of the energy influx.

Different methods are established for determination the energy flux at plasmatechnological processes: for example by measuring the temperature difference generated on a substrate $[1,6]$, by measuring the increasing temperature in the centre of a membrane being exposed to an energy flux and clamped and cooled by a guard ring $[4,7]$, or by recording the temporal heating and cooling temperature course of a dummy substrate $[5,8,9]$.

These measuring methods often produce problems by measurement errors, caused by undesirable heat transport processes that are difficult to control. This concerns for example the heat conduction of the probe and/or the change of the heat capacity by coating the probe. Furthermore, the probe has to be calibrated by an energy source of known intensity, which is associated with uncertainty again. For example, the frequently unknown environmental conditions during calibration usually differ and changing reflexion and emission properties of the thermal probe occur.

Ellmer et al. carried out measurements with a Gardon sensor and tried to minimize the errors by blackening the probe [4,7]. However, the calibration results deviated strongly from the expected value because of the heat conduction of the thermal probe. [4] Even through calibrating the passive thermal probe, errors of at least $20 \%$ were estimated [5,10,11].

Stahl et al. have calibrated passive probes by an electron beam to minimize the measurement error [12]. Furthermore, Bornholdt et al. used a transient method for measurements with a passive thermal probe [13]. In addition, Ellmer et al. investigated a method for calibrating their calorimetric sensor by charged particles emitted from the plasma [14].

\section{Theoretical considerations and principle of measurement}

The problems of calibration were the reason for investigating other methods to measure at a constant thermal balance where heat conduction should not influence the results and calibration is not required.

The following principle of the active thermal probe is applied: likewise as a "dummy substrate" the probe is used to determine the energy flux to a given area. The probe is placed in the region of the plasma where the energy influx needs to be measured.

At the beginning, the probe is heated until the temperature balance reaches the equilibrium temperature $T_{\text {equ }}$ without any plasma exposure.

The heating energy $\left.J_{h}\right|_{\text {cool }}$ is supplied as needed, e.g. by an electrical heater. The incoming heat flux received at the probe $\left(Q_{i n}\right)$ and the outgoing heat flux $\left(Q_{o u t}\right)$ in this "cold" phase (without plasma) are

$$
\begin{aligned}
\left.Q_{\text {in }}\right|_{\text {cool }} & =\int_{A}\left(\left.J_{h}\right|_{\text {cool }}+J_{\text {env }}\left(T_{\text {env }}\right)\right) d A \\
\left.Q_{\text {out }}\right|_{\text {cool }} & =\int_{A}\left(J_{\text {rad }}\left(T_{\text {equ }}\right)+J_{\text {con }}\left(T_{\text {equ }}-T_{\text {car }}\right)\right) d A
\end{aligned}
$$

Here $J_{e n v}$ is the heat conduction from the environment, $T_{e n v}$ the environmental temperature, $J_{\text {rad }}$ the heat loss at the probe by radiation, $J_{c o n}$ the heat flux from and to the probe generated by heat conduction, $T_{c a r}$ the temperature of the probe holder or 
the connection cable and $A$ is the probe area. Since thermal balance is assumed, the following equation must be valid

$$
\left.Q_{\text {in }}\right|_{\text {cool }}=\left.Q_{\text {out }}\right|_{\text {cool }}
$$

and, thus,

$$
\left.J_{h}\right|_{\text {cool }}=J_{\text {rad }}\left(T_{\text {equ }}\right)+J_{\text {con }}\left(T_{\text {equ }}-T_{\text {car }}\right)-J_{\text {env }}\left(T_{\text {env }}\right)
$$

The probe is conditioned by adjusting the temperature balance and determining the required energy input $\left.J_{h}\right|_{\text {cool }}$.

In the case of an additional energy flux $J_{i n}$ from the plasma, the temperature rises and the thermal balance is disturbed. This effect is counteracted by lowering the input heat $\Delta J_{h}$ to a certain amount until the temperature balance $T_{e q u}$ is achieved again. To maintain this state, the energy $\left.J_{h}\right|_{\text {cool }}$ becomes redundant and is replaced by a lower energy.

$$
\left.J_{h}\right|_{\text {heat }}=\left.J_{h}\right|_{\text {cool }}-\Delta J_{h},
$$

since some of the heat loss is compensated by the energy flux. It can easily be demonstrated that now the heating power is

$$
\left.J_{h}\right|_{\text {heat }}=-J_{\text {gain }}-J_{Q}-J_{\text {env }}\left(T_{\text {env }}\right)+J_{\text {rad }}\left(T_{\text {equ }}\right)+J_{\text {con }}\left(T_{\text {equ }}-T_{\text {car }}\right)
$$

Here, $J_{Q}$ is the energy influx at the probe, which is produced by the heat radiation of the source and $J_{\text {gain }}$ is the energy influx at the probe produced by the plasma process (without the heat radiation of the source).

If this relation together with equation (4) is combined with equation (5), the actual energy $J_{i n}$ at the probe can be obtained at the equilibrium temperature $T_{\text {equ }}$ :

$$
J_{\text {in }}=\Delta J_{h}=J_{\text {gain }}+\left.J_{Q} \quad\right|_{\text {Tequ }}
$$

There are no terms in equation (7) which depend on probe temperature, environmental temperature or the temperature of the probe holder, respectively.

Furthermore, the measured energy is independent of the heat capacity of the probe. That is an important fact because now the determination of the energy by calibrating a known radiation source is not necessary. Another advantage is the independence of the measurement on the heat conduction $J_{c o n}$ along the probe holder.

Because the measured energy influx is, in principle, the disturbing variable of the temperature balance, the probe becomes less sensitive due to higher heat loss through the holder and connection cable. The compensation of this disturbance is directly proportional to the efficiency of the disturbance, i.e. all other heat flows in equation (6) which influence the heat flux $\left.J_{h}\right|_{\text {heat }}$. If the thermal radiation $J_{\text {rad }}$ of the probe or the heat conduction $J_{c o n}$ through the connecting cables is high, all other terms remain ineffective.

These conclusions can be found in the given balance of equation 6: The heating power $\left.J_{h}\right|_{\text {heat }}$ cannot be negative. Therefore, considering equation (5) the heating power must always be set higher than the energy flux to be measured. The sensitivity and the maximum of the measurable energy flux can be influenced by the geometry of the probe holder. 
For accurate measurements of the energy influx all incoming and outgoing heating fluxes to the probe which are not involved in measurement value have to be assumed constant in time.

This requires specifically constructed probe holders. They contain one or more heated zones, which are set to a given constant operation temperature close to the probe temperature. Thus, the heat fluxes between holder and probe are zero or constant.

A similar configuration is used for the construction of the double probe. Two identical probes are bonded together with their back sides. If both probes are at the same temperature, the heating flux between them is zero. Hence, each probe detects only the incoming energy flux from one half-space. By rotation of the probe this can be used to obtain a "heating radar image" of the environment.

In particular, the ability for continuous measurement is a great advantage of the active probe. After reaching the temperature balance, any change of energy influx from plasma is registered and the temperature balance is restored again. However, setting the temperature balance level as well as the energy influx measurement occurs with a time delay. Note that only the change of the energy influx can be measured and be used as a control variable at the same time when used in process controlling.

\section{Configuration of the probe and measurement procedure}

A specially constructed PT100-cell heated by an electrical current, whose temperature can be measured and controlled by a supplied external power is exposed to the energy flux of the plasma. The supplied power and the temperature can be calculated from the potential difference and the current. The probe is connected through a shunt to the output of an amplifier which is controlled by a digital-analog converter. Customised software is installed to filter the readings, control the temperature and calculate the energy influx. For thermal isolation, a second heating is located between the sensor area and the holder, which works in the same way and is set to the same temperature as the sensor. This is necessary to ensure that during the measurement the energy fluxes between holder and probe are zero or constant in time. This is important for the validity of equation 7 .

Figure 1 shows a photograph of a sensor with a size of about $7 \times 10 \mathrm{~mm}$.

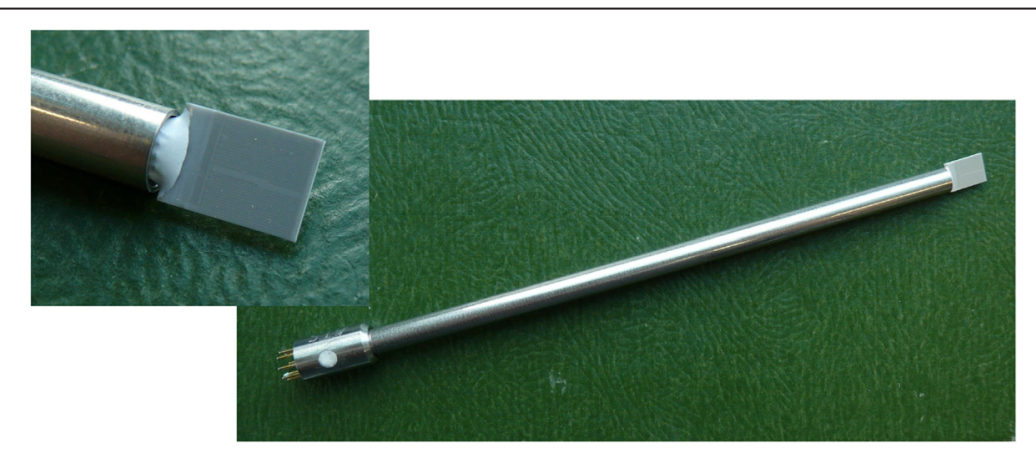

Figure 1 Active Probe, e.g. Pt100-cell on the manipulator arm. 
The procedure stores the value of power necessary to maintain the temperature balance level at set-point temperature without energy influx and controls the temperature balance level by analysing the value and the changing rate of the probe temperature. Also it calculates the difference between the power currently needed and the power needed without energy influx from plasma. It displays directly if measurement starts the incoming energy influx at the probe in $\mathrm{mW}$.

\section{Experimental results}

Test measurements were obtained by a prototype of the active probe in a plasma chamber at a pressure of $0.04 \mathrm{~Pa}$. The first test should show if the active thermal probe can provide reliable and repeatable data of the energy influx in a plasma environment. For that reason an ion beam is quite suitable because it is a very constant energy source without coating the probe.

An ion beam source at beam voltage of $500 \mathrm{~V}$ and with beam diameter of $160 \mathrm{~mm}$ was used at a distance of $225 \mathrm{~mm}$ in front of the probe. Typical measurements are shown in Figure 2, e.g. the temporal course of the input heating power at the probe and the resulting temperature versus time. After switching the energy flux (ion beam) at $\mathrm{t}=300 \mathrm{~s}$ the probe temperature increases shortly from $343^{\circ} \mathrm{C}$ to $355^{\circ} \mathrm{C}$ and the control unit responds by decreasing the heating power from about $580 \mathrm{~mW}$ to about $40 \mathrm{~mW}$. After a certain time the temperature balance level $\left(343^{\circ} \mathrm{C}\right)$ is reached again. At this point the input power is approximately $540 \mathrm{~mW}$ lower then at the "plasma off" point (at the time from 100 to $300 \mathrm{~s}$ ) which is equivalent to the energy influx from the plasma ion source to the probe. After switching-off the source (at $\mathrm{t}=950 \mathrm{~s}$ ), the probe temperature decreases shortly but reaches its set point again after about $10 \mathrm{sec}$.

The energy influx at the probe can be measured with an accuracy of approximately 1 $\mathrm{mW} / \mathrm{cm}^{2}$, which has been confirmed by the fluctuations of the used prototype. Smaller changes in the energy influx are not measurable because of the stochastic fluctuations of the data.

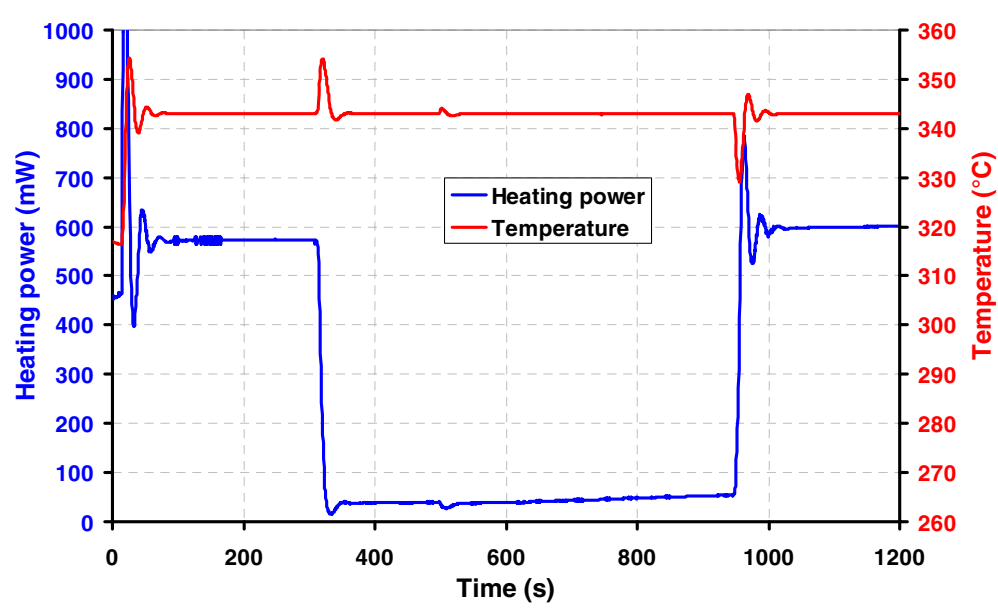

Figure 2 Temporal behaviour of temperature and heating power at the active probe during operation of an ion beam source $[15,16]$. 


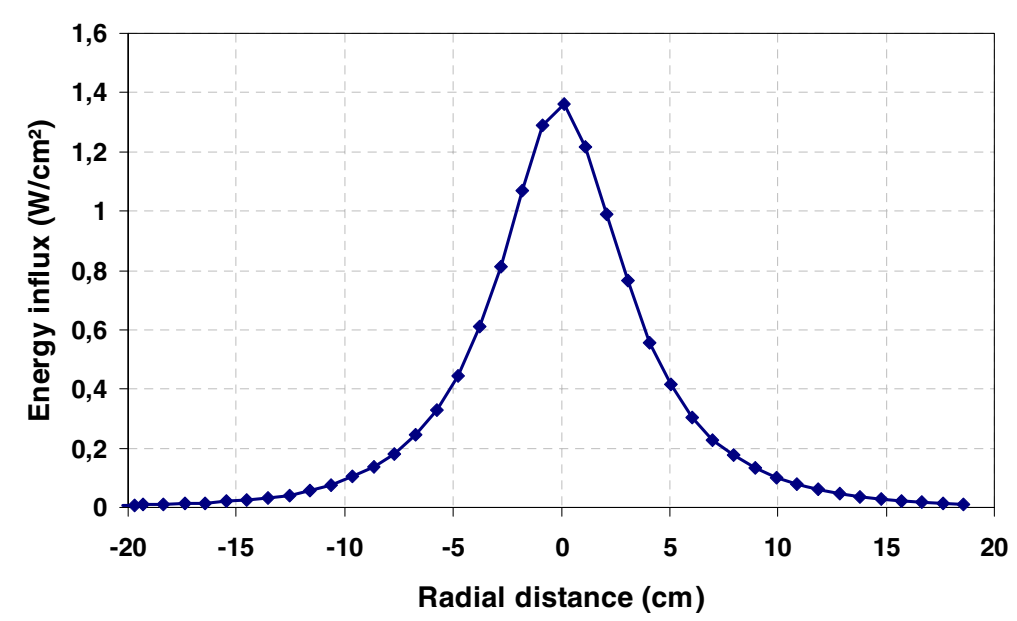

Figure 3 Radial profile of the energy influx of the ion beam source $\emptyset 160 \mathrm{~mm}$, based on measurements as shown in Figure 2.

The time needed to reach the temperature balance level of the probe was in the range of $30 \mathrm{~s}$ which may be for many applications, especially for controlling and regulating long-time plasma processes, an acceptable value. The time for reaching the equilibrium depends on the heat capacity of the sensor and the quality of the temperature control technique.

However, by the process control the reaction time of the probe can be much shorter. A dysfunction of the process can already be detected by small variations of the probe heating power. That means, the temporal gradient of the heating power can be an indicator for the process regulation. Typical dimensions of the probe (from $2 \times 2 \mathrm{~mm}$ to $7 \times 7 \mathrm{~mm}$ ) allow for measurements with sufficient spatial resolutions.

Finally, the result of the first test was that the active thermal probe is suited to provide reliable data of the energy influx. As a useful example the radial profile of the energy influx in the ion beam of the described source was measured and is shown in Figure 3. Although the beam diameter is $160 \mathrm{~mm}$ in a distance of $225 \mathrm{~mm}$ there is a broader profile due to divergence effect. By means of this measured profile the symmetry of the ion source can be evaluated.

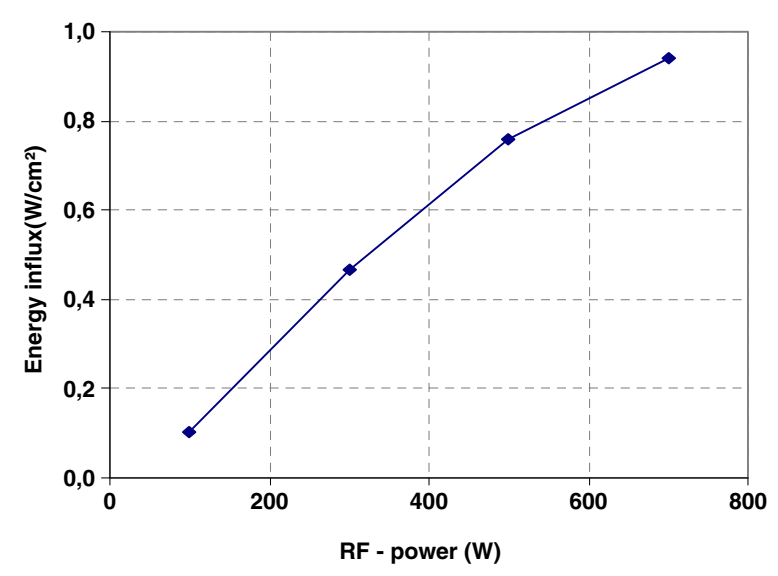

Figure 4 Energy influx versus RF power in an inductively coupled RF plasma. 


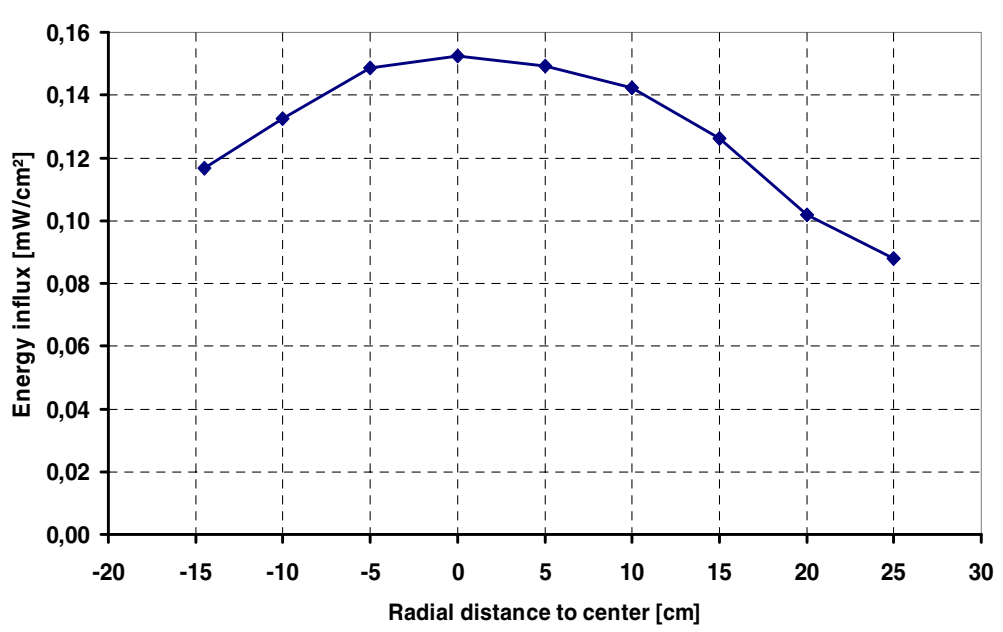

Figure 5 Radial energy influx profile of an APS source.

Additional test measurements have been performed in an inductively coupled rf-plasma and a dc-plasma source (APS, so-called advanced plasma source [17]). These experiments should prove if the electromagnetic field of the plasma disturbs the measurements. For the inductively coupled rf-plasma in argon (pressure 0.5 Pa, rf-power $300 \mathrm{~W}$ ) used for plasma etching the dependence of the energy influx on the rf-power is shown in Figure 4. For the APS source (bias voltage $133 \mathrm{~V}$, power $7.3 \mathrm{~kW}$, pressure $0.02 \mathrm{~Pa}$ ) a radial profile has been measured, too, in order to illustrate the applicability of the sensor, see Figure 5. Both plasma sources are commonly used in coating devices for the production of optical films. Also a measurement in such as plasma environment is possible.

In thin film deposition processes the probe may be coated itself - and its behaviour under such conditions is of special interest. For this purpose, the probe was alternately coated by copper and titanium. The results are shown in Figure 6. The coating experiments were carried out in a chamber with two magnetrons at a pressure of $0,5 \mathrm{~Pa}$, an argon flux of $100 \mathrm{sccm}$, a magnetron power of $1000 \mathrm{~W}$ and a probe temperature of about $320^{\circ} \mathrm{C}$. Before the measurements the probe was coated with a titanium layer. At $250 \mathrm{~s}$ (see Figure 6) the copper coating process starts. It could be observed that the heating power of the probe is decreased by the incoming energy influx. Since the reflection coefficient of copper is lower then in the case of titanium the incoming energy increases as the copper film is growing. This effect causes the decrease of the heating

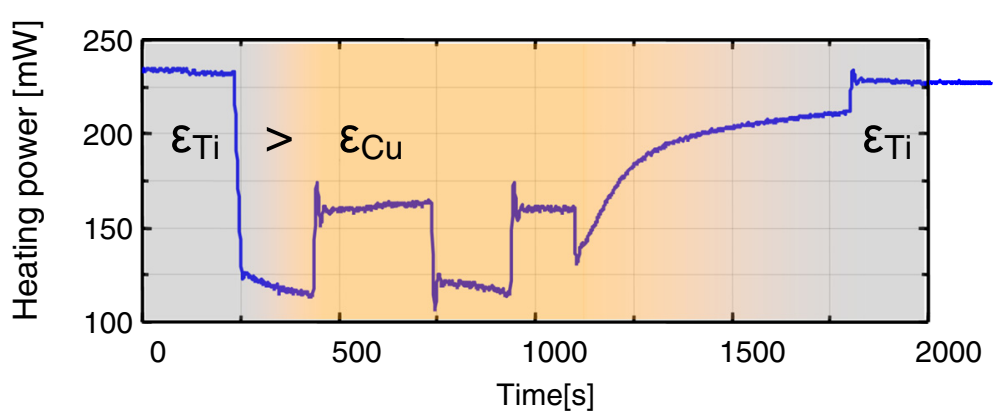

Figure 6 Heating power of the probe by alternate coating: titanium-copper-titanium. 


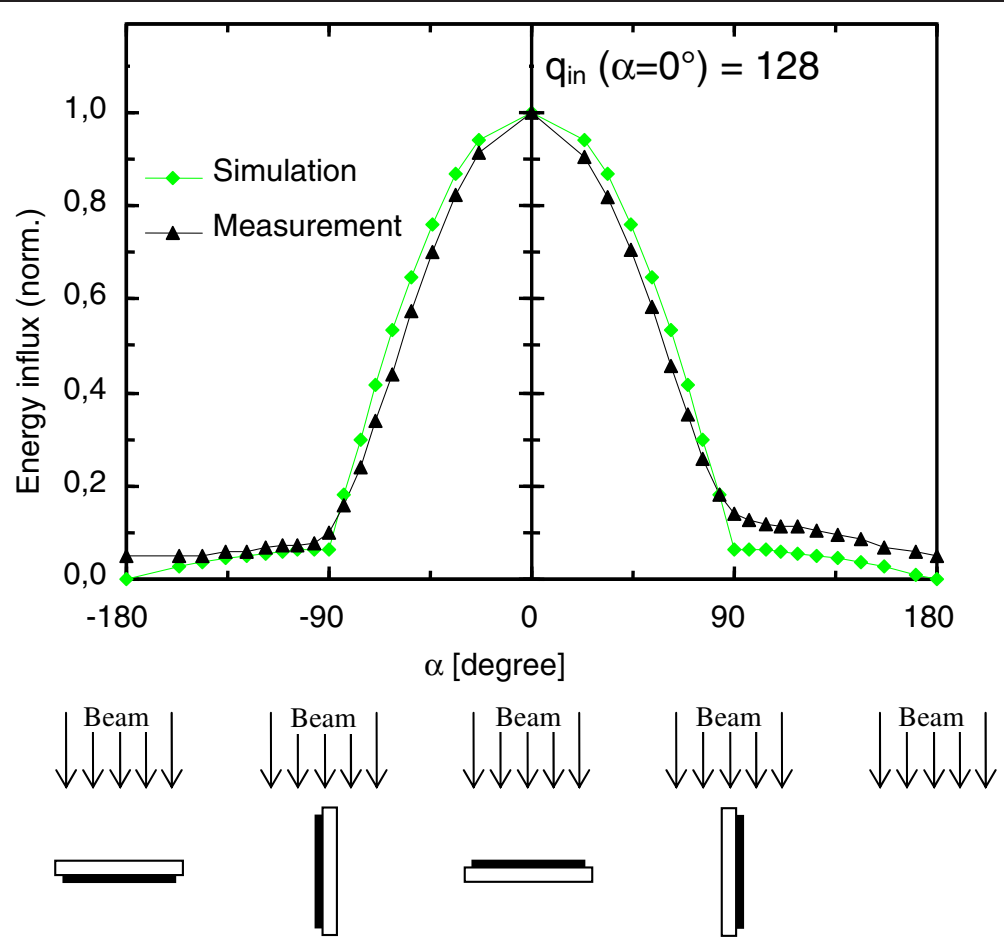

Figure 7 Energy influx versus probe angle to the ion beam axis.

power in the time interval $t=250 \mathrm{~s}$ to $450 \mathrm{~s}$ (see Figure 6). After closing of the aperture, the energy flux is interrupted. But the base level at $230 \mathrm{~mW}$ is not reached again. Assuming that the probe temperature is constant. This observation means, that the radiation loss of the copper layer has significantly decreased. The initial level (source switched off) of about $160 \mathrm{~mW}$ is also conserved after additional coatings with copper $(\mathrm{t}=500 \mathrm{~s}$ to $1200 \mathrm{~s})$. At $\mathrm{t}=1200 \mathrm{~s}$ the titanium coating starts again. The cumulative coating with titanium changes the radiation coefficient of the probe surface again. The radiation losses increase and the heating power of the probe increases too $(t=1200 \mathrm{~s}$ until $1600 \mathrm{~s})$. If a welded layer is produced, then the heating power will be constant. After closing of the aperture (1900 s) the coating process is finished and the base level of the experiment is almost reached.

Hence, the application of the thermal probe at different coating processes is possible. However, the coating material should be not changed during the measurement.

A quite interesting benefit of the probe is the application as a double probe. By means of such a probe setup the direction of the incoming energy influx can be detected. Two identical probes are bonded by their back sides. The practical experience has shown that all control cycles on the probe chips operate accurately. Obviously, the thermal resistance between both chips is sufficiently high for the thermal decoupling.

With this configuration angle-resolved measurements with an ion source (type Vecco ALS340) has been performed. The process parameters were as follows: pressure at 0,4 pa argon with $10 \%$ oxygen, a beam voltage of $2 \mathrm{kV}$ and a beam current at $415 \mathrm{~mA}$. The probe is located in front of the ion source in a distance of $30 \mathrm{~cm}$. During the 


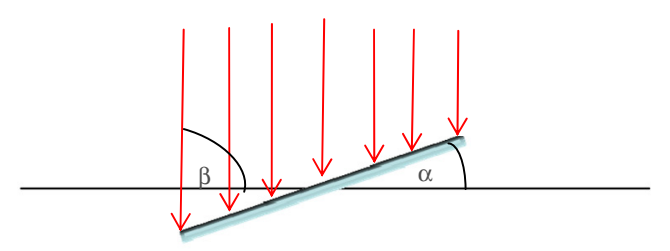

Energy influx versus probe angle to beam axis

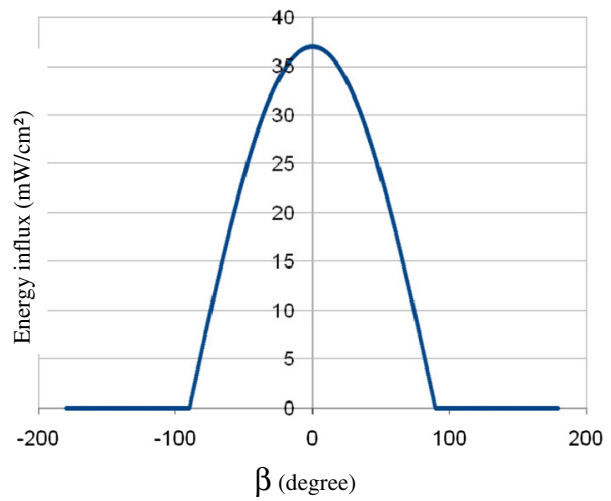

Energy influx versus radiation angle to probe level

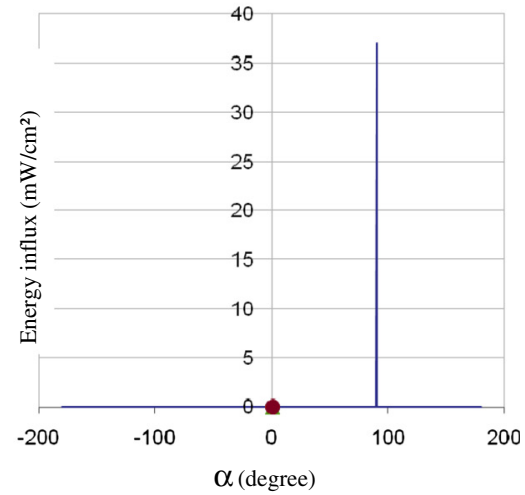

Figure 8 Energy influx versus probe angle to the beam axis and versus radiation angle.

measurement the probe was rotated around the roll-axis. The data in Figure 7 shows the expected behaviour which depends on the cosine of the probe angle to the beam axis. Also a calculation of the angular values is included at angles from $90^{\circ}$ to $180^{\circ}$ the probe edges are exposed by the ion beam.

The observed behavior of the double probe indicates clearly that there is no heat flux from the back side to the measurement area on the front side of the probe.

By the use of the Fourier transform the dependence of the energy influx on the probe angle to the beam axis is converted to the dependence on the angle between probe level and the direction of the incoming energy influx. The result is shown in Figure 8.

Thus, the double probe can be used to obtain a "heating radar image" of the probe environment.

\section{Conclusion}

By means of the described prototype it could be verified that the principle of an active heated thermal probe is applicable for the determination of the energy influx in several plasma-technological processes. The principle is based on the decrease of the external input heating power at the probe, which is needed to compensate the incoming energy flux from the plasma. The attained sensitivity almost reaches the level of passive probes. Further optimisation in terms of sensitivity and time resolution should be made for broader applications. An increase in sensitivity while measuring lower energy influxes could be achieved by minimising the heat capacity of the probe and improving the temperature regulation. For special applications, a miniaturised version of the probe in the form of a microchip is conceivable, which would lead to a considerable decrease in the probe's response time. 
Authors' contributions

All authors read and approved the final manuscript.

\section{Acknowledgment}

We like to thank the AIF for supporting the investigations in the frame of the ZIM-project VP2345701DF9 (E-impact).

\section{Author details}

${ }^{1}$ Institut für Experimentelle und Angewandte Physik, Leibnizstr. 19, Kiel D-24098, Germany. ${ }^{2}$ Formerly Institut für

Plasmaforschung und Technologie, Felix-Hausdorff-Str. 2, Greifswald D-17489, Germany.

Received: 25 September 2014 Accepted: 4 February 2015

Published online: 24 February 2015

\section{References}

1. Bornholdt S, Peter T, Strunskus T, Zaporojtchenko V, Faupel F, Kersten H. IL-6 release after intestinal ischemia/reperfusion in rats is under partial control of TNF. Surf Coat Techn. 2011;205:388-92.

2. Steffen $\mathrm{H}$, Kersten $\mathrm{H}$, Wulff $\mathrm{H}$. Investigation of the energy transfer to the substrate during titanium deposition in a hollow cathode arc. J Vac Sci Technol A. 1994;12:2780.

3. Kersten H, Rohde D, Berndt J, Deutsch H, Hippler R. Investigations on the energy influx at plasma processes by means of a simple thermal probe. Thin Solid Films. 2000;377-378:585-91.

4. Cormier PA, Stahl M, Thomann AL, Dussart R, Wolter M, Semmar N, et al. On the measurement of energy fluxes in plasmas using a calorimetric probe and a thermopile sensor. JPhysD: Appl Phys. 2010;43:465201.

5. Lundin D, Stahl M, Kersten H, Helmersson U. Energy flux measurements in high power impulse magnetron sputtering. J Phys D Appl Phys. 2009:42:185202.

6. Ellmer K, Mientus R. Calorimetric measurements with a heat flux transducer of the total power influx onto a substrate during magnetron sputtering. Surf Coat Techn. 1999:116-119:1102-6.

7. Gardon R. An Instrument for the direct measurement of intens thermal radiation. Rev Sci Instrum. 1953;24:366

8. Thornton JA. Substrate heating in cylindrical magnetron sputtering sources. Thin Solid Films. 1978:54:23.

9. Kersten $H$, Deutsch $H$, Steffen $H$, Kroesen GMW, Hippler R. The energy balance at substrate surfaces during plasma processing. Vacuum. 2001;63:385-431.

10. R.Wiese. Neue Methoden der Diagnostik von Plasmaquellen, Dissertation 2007, Library Ernst-Moritz-Arndt Universität Greifswald

11. Wiese R, Kersten H. Einsatz einer aktiven Thermosonde zur Diagnostik von Prozessplasmen. Fachzeitschrift Galvanotechjnik. 2008;6:1502-7.

12. Stahl M, Trottenberg T, Kersten H. A calorimetric probe for plasma diagnostics. Rev Sci Instrum. 2010;81:023504.

13. Bornholdt S, Kersten H. Transient calorimetric diagnostics for plasma processing. Eur Phys J D. 2013;67:176

14. Wendt R, Ellmer K, Wiesemann K. Thermal power at a substrate during ZnO:Al thin film deposition in a planar magnetron sputtering system. J Appl Phys. 1997;85:2115-22.

15. Zeuner M, Scholze F, Neumann H, Chassé T, Otto G, Roth D, et al. A unique ECR broad beam source for thin film processing. Surf Coat Technol. 2001;142-144:11-20.

16. Wiese R, Kersten H, Wiese G, Häckel M. Aktive Thermosonde zur Messung des Energieeinstromes. Vakuum in Forschung und Praxis. 2011;23:20-3.

17. Schroeder B, Peter R, Harhausen J, Ohl A. Modelling and simulation of the advanced plasma source. J Appl Phys 2011;110:043305.

\section{Submit your manuscript to a SpringerOpen ${ }^{\circ}$ journal and benefit from:}

- Convenient online submission

Rigorous peer review

- Immediate publication on acceptance

- Open access: articles freely available online

- High visibility within the field

- Retaining the copyright to your article 